\title{
La revolución cultural y el marxismo
}

\author{
Vania Bambirra es profesora de sociologia e investigadora del Centro \\ de Estudios Socio-Económicos de la Facultad de Ciencias Económicas \\ de la Universidad de Chile.
}

La China Contemporánea: el Kewes S. Karol. México: Siglo otro lado del río. Tomos 1 y $\cdot 11 . \quad V e i n t i u n o$, Editores, 1967.

Edgar Snow. México: Fondo de China entra en escena. Roger Cultura Económica, 1965.

China: el otro comunismo. de Mirasol, 1967.

"China se encuentra en el centro de la politica mundial $y$ demasiadas cosas dependen de ella para que podamos contentamos con condenarla o con desconocerla. Lo oportuno, más bien, seria intentar comprenderla".

Kewes S. Karol

1. LAS DIFICULTADES QUE SE ANTEPONEN A LA COMPRENSIÓN DEL FENÓMENO CHINO

La Revolución Cultural ha generado muchas especulaciones $y$ controversias ${ }^{1}$. Pero pocos fueron los que en el occidente, lo mismo que entre los marxistas, lograron comprender algo de su verdadero carácter y de sús cónsecuencias. Esa perplejidad generalizada revela cuán grandes son las dificultades existentes para la comprensión de China. Trataremos de analizar las principales dificuiltades, que son las generadas por la visión occidental y por el estancamiento del marxismo.

\section{a) La dificullad causada por las visiones occidenlales}

Esta está determinada por cuatro factores:
a) aislamiento
b) desconocimiento
c) sentido de superioridad
d) etapas distintas en el proceso de organización social.

\footnotetext{
${ }^{1}$ Este articulo no tiene la pretensión de suministrar una interpretación definitiva de la Revolución Cultural. No somos especialistas en los asuntos chinos y además faltan datos fundamentales que no tienen divulgación en el occidente. Lo que intentamos hacer fue tomarla como punto de partida para plantear algunas hipótesis generales que quizás si fueren desurrolladas podrian ampliar la base teórica para la comprensión de muchos de sus problemas y de los que vive hoy el campo socialista.
} 
Como bien lo analiza Edgar Snow, "China se desarrolló casi totalmente aislada de Occidente", e intenta explicar ese aislamiento por factores geográficos y culturales. Dejando de lado los primeros, que en la época contemporánea han perdido gran parte de su importancia, nos vamos a detener en los factores culturales.

Es indispensable recordar que la sociedad china tiene tradiciones culturales milenarias, radicalmente distintas de las occidentales. Aunque hov dia China intente deshacerse de ellas (porque considera que esa cultura es adecuada a los intereses de dominación de la sociedad que la genera y por lo tanto debe desaparecer junto con esta), sus marcas han sido hondas en el pueblo chino y si se intenta comprenderlo, no se puede prescindir de ellas. Tomemos por ejemplo el ideograma: aunque actualmente tienda a simplificarse considerablemente, su aprendizaje resulta muy arduo para un occidental. Ahora bien, el ideograma parece que refleja no solamente una manera de expresar sino, más aún, una forma de pensar sintéticamente. El occidental está más acostumbrado a pensar al expresárse, mientras los chinos meditan más antes de expresarse. Por eso su forma de razonamiento nos parece muchas veces mera simplificación, en la medida que no logramos captar sus diferenciaciones profundas. Esto tiende a acentuarse después de la Revolución Cultural al aumentar la preocupación de parte del gobierno por razonar en forma didáctica, comprensible para el pueblo.

El aislamiento es agravado por la ignorancia de la cultura china. Entre los occidentales que hicieron estudios históricos, raros son los que conocieron un poco más allá de la historia de la civilización occidental. El oriente era como una mancha olvidada del mapa. Cierto es que, como destaca Edgar Snow, por parte de los chinos hubo, por lo menos hasta la revolución, una cierta complicidad pues también miraban al resto del mundo con una óptica oriental, $y$ por a mbos lados existió la barrera del sentido de superioridad ${ }^{2}$.

Pero, de parte de occidente, en la medida en que el desarrollo industrial se verificó primero, ese sentido de superioridad sirvió como racionalización de la dominación imperialista. Es fundamentalmente la necesidad de expansión imperialista la que va a agudizar hasta las últimas consecuencias lo que llamamos sentido de superioridad, la que va a posibilitar la agudización de la visión occidental para justificar que hay que occidentalizar el oriente, 0 sea, hay que posibilitar la dominación colonial-imperialista, hay que destruir las culturas "atrasadas" y hacer posible la penetración del modo de producción capitalista. De aqui que cualquiera que intente comprender China tiene que realizar un esfuerzo muy grande para deshacerse de esta visión occidental, que es, en el fondo, sinónimo de visión imperialista.

\footnotetext{
${ }^{1}$ La obra de Roger Pelissier, presenta una ilustrativa serie de documentos desde 1839 , muchos de los cuales sirveri como comprobación del sentido de superioridad chino frente al occidente.
} 
El mismo razonamiento sólo seria válido, en parte, para Japón. Porque Japón no resistió a la penetración imperialista como China. Japón no hizo una profunda revolución social. A la inversa, se "occidentalizó" en la medida en que la sociedad japonesa permitió la penetración imperialista y se transformó a si mismo en una potencia imperialista. Claro es que el capitalismo japonés tiene caracteristicas muy propias que merecerian un análisis especial que no corresponde hacer ahora.

Por eso deciamos que los estudiosos occidentales de China no logran comprenderla. La excelente obra de Kewes S. Karol es un ejemplo de esto. Karol no es un especialista en asuntos chinos, sino un intelectual interesado en China porque está convencido del importante papel que juega y ha de jugar más aún en el escenario mundial. Su obra proporciona un excelente panorama de la China contemporánea, no sólo porque estuvo allá poco antes del comienzo de la Revolución Cultural, sino también porque es un análisis honesto y objetivo de lo que le fue posible conocer de China. Pero en toda la obra, aunque mantiene una actitud favorable hacia China, se siente algo de perplejidad y se manifiesta la incomprensión de una mentalidad occidental. Esto se nota secundariamente en las dudas que expresa Karol sobre la sinceridad de los relatos, hecho en que hasta un cierto punto puede tener razón, pero sobre todo cuando empieza a comparar los valores chinos con los occidentales; por ejemplo, cuando no logra captar el contenido profundo que tiene para el pueblo el héroe Lei Feng. Karol reconoce que ese héroe era una extraordinaria figura humana, pero como sus acciones no tuvieron nada de espectacular, Karol encuentra ingenua su elevación a esa categoria.

En realidad (y ahora comenzamos la discusión del último y más decisivo obstáculo que señalamos para la comprensión de China), lo que ocurre es que China ya alcanzó un estudio más avanzado de su organización social y, por lo tanto, ésta constituye una barrera muy grande hacia su comprensión. Karol, por ejemplo, debido a la gran objetividad con la cual se esfuerza por comprender China, llega muy cerca de un nivel de comprensión de lo que está ocurriendo en realidad, pero se queda ahi, entre el limite de una visual occidental y la realidad económico-social china:.

Sólo poniéndose en la perspectiva del proceso de avance rápido hacia el comunismo en China, es que se puede comprender el significado de un héroe como Lei Feng. Porque sólo asi puede comprenderse por qué en la China contemporánea es más importante que nada, la fraternidad, la humildad, la solidaridad y la valorización del trabajo. Porque son estos valores los que realmente se oponen al individualismo, la prepotencia y la

\footnotetext{
1.... es demasiado pronto para prejuzgar acerca del porvenir de la sociedad igualitarista de China. Por el momento, sin embargo, representa ya una realidad, que no tiene precedentes y que no es fácil que comprendan los occidentales aún cuando tengan formación marxista". K.S.Karal, p. 379
} 
alienación que en China ya empiezan a ser parte del museo de un régimen -social que está siendo definitiva e irreversiblemente derrocado en un sector (¡qué es bien grande!) del mundo. De lo que se trata ahora es de enfrentar la realidad cotidiana de la contracción del comunismo, aunque esa realidad puede parecer en el fondo aburrida, aún para quien se considera muy revolucionario. De hecho, aún el más izquierdista, no está acostumbrado a meditar en lo radicalmente nueva y distinta que será la experiencia comunista. Uno está demasiado impregnado por el modo de vida occidental. Ya decia un prominente sociólogo brasileño, después de volvar de China: "China es fabulosa pero es terriblemente aburrida". En parte, ese aburrimiento podria estar reflejando una inadaptación frente a lo que es chino especificamente, pero en parte reflejaria, sin que se diese cuenta, una falta de preparación para adaptarse al nuevo régimen y sus implicaciones en la vida personal de cada uno. Hay que distinguir entre las dos cosas.

\section{b) La dificultad causada por el estancamienlo del marxismo}

Como señalan muy bien Sweezy y Baran "obras importantes de ciencia social marxista hạ sido pocas en los últimos años. Con demasiada frecuencia, los marxistas se contentaron con la repetición de formulaciones ya familiares, como si nada realmente nuevo hubiese ocurrido desde los dias de Marx y Engels, o de Lenin por lo menos. Consecuentemente, los marxistas no pudieron explicar evoluciones importantes ni siquiera a veces reconocerles su existencia"l.

Pero el estancamiento del marxismo no se debe a limitaciones inherentes a su metodologia sino a su no aplicación o a la mala utilización del método dialéctico. Se debe a varios factores entre los cuales quizá el más importante haya sido la existencia del stalinismo y su influencia en el movimiento revolucionario mundial. El hecho es que el capitalismo asumió nuevas formas (el capitalismo monopólico en los centros hégemónicos y el capitalismo dependiente en los paises periféricos) que sólo ahora comienzan a ser estudiados satisfactoriamente. Lo mismo se puede decir del socialismo en cuanto régimen social, como también de la existencia del campo socialista: que ambos fueron muy poco analizados. Los problemas planteados por el desarrollo del socialismo desafian la comprensión de la Intelectualidad y ponen en el orden del dia la necesidad de hacer avanzar el pensamiento marxista.

En la fase en que se encuentra el socialismo en China es necesario saber enfrentar con la metodologia marxista a problemas completamente nuevos, áún no tratados. Ya decia Marx: "la humanidad sólo se plantea problemas que puede resolver". Ahora, el pensamiento marxista tiene que enfrentar problemas completamente modernos que quedan sin contestación e impiden

\footnotetext{
${ }^{1}$ Capilalismo Monopolista. Zahar Editores. Rio de.Janeiro, 19006, p. 13. (La traducción es nuestra).
} 
la comprensión de las fases de la revolucion. Trataremos de enunciar rápidamente sólo algunos de estos problemas que, a nuestro juicio, deben ser objeto del esfuerzo intelectual marxista, tomando como punto de referencia a China.

\section{La necesidad de definir lo que es el socialismo}

Los clásicos se detuvieron principalmente en el estudio del capitalismo; sobre el socialismo hicieron apenas algunos enunciados generales, que aunque contenian intuiciones geniales no sirven para aclarar el contenido complejo del socialismo como práctica social. Por eso es licito, necesario y urgente, un siglo después de "El Capital", preguntarnos: ¿qué es el socialismo?

Para nosotros el socialismo no llega a configurarse como un sistema social propiamente tal sino como un régimen de transición hacia el comunismo, de la misma forma que los regimenés que brotaron con la revolución comercial (el absolutismo monárquico) fueron una transición del feudalismo hacia una formación social tipicamente capitalista.

El carácter de transición del régimen socialista está dado por la coexistencia, aunque en continuo choque, de dos modos de producción y de dos superestructuras: la anterior que se derrumba, y la nueva que se instaura. De este choque tiene necesariamente que resultar la liquidación de una o de otra; sea a través de una involución a formas presocialistas ${ }^{1}$, sea a través de un avance hacia una organización social y económica completamente nueva. Asi, en el fondo, el dilema no.es capitalismo versus socialismo, sino capitalismo versus comunismo.

El carácter de transición del socialismo proviene de sus contradicciones entre lo nuevo y lo viejo. Por ejemplo, la burocracia, que hasta hoy no ha sido suficientemente analizada, es, al mismo tiempo, una necesidad del socialismo, aunque en su esencia sea un elemento antiproletario $y$ tienda mucho más a conservar los caracteres del viejo orden social.

La idea de que el socialismo es un régimen de transición está ya en los clásicos, especialmente en "El Estado y la Revolución" de Lenin. De alli puede extraerse la concepción de que ese régimen sólo se justifica en dos aspectos:

1. En la medida en que sus tareas son destructivas, es decir que se trata de destruir los aparatos de dominación burguesa, como el ejército, el aparato estatal y las bases económicas de dominación de la burguesia. Es un régimen en el cual todavia sobrevive la dominación de clase que es la dictadura del proletariado (por lo menos teóricamente, pues, en la práctica, en muchos paises, esa dominación resultó ser antes que nada una dominación de sectores burocráticos)

\footnotetext{
${ }^{1}$ Es necesario aclarar que se trata de una involución hacia un capitalismo de Estado con caracteristicas peculiares, de tipo estatal y altamente burocratizado; en el cual la explotación del excedente económico se haria indirectamente, por intermedio de un complejo aparato gubernamental.
} 
2. A través de tareas constructivas, es decir, del establecimiento de las bases económicas, sociales y culturales que posibiliten la instauración de un régimen tipicamente comunista.

Asi tenemos, por un lado, tareas destructivas y por otro tareas preparatorias: este es el proceso de la revolución socialista que resulta tan lleno de contradicciones que ella misma no puede resolver, y que le imprimen un carácter transitorio. Ese proceso puede ser largo o corto. Depende de las condiciones internas de cada pais y del contexto mundial. Si en la uRss este proceso ya lleva medio siglo, en Estados Unidos podria verificarse en mucho menos tiempo porque se estaria poniendo en jaque el sistema de dominación imperialista mundial.

La necesidad de redefinir lo que es el comunismo y definir cuales son las leyes generales de su movimiento

Lo que se entiende por comunismo es un régimen donde no habria más luchas de clases ni por lo tanto Estado; donde existiria el reino de la abundancia; donde no existiria más la división entre el trabajo manual e intelectual; donde el hombre lograria liberarse de la alienación y ser realmente libre. Además, el comunismo sólo seria posible como sistema internacional. Esa fue la manera como los clásicos concibieron el comunismo. Constituia una respuesta teórica a problemas que todavia no habian sido planteados por la práctica $y$, por lo tanto, no pasaban de ser especulaciones generales. Marx y Engels estaban preocupados con la comprensión del capitalismo y Lenin nunca analizó nada que no tuviera una aplicación inmediata; sus obras estuvieron siempre marcadas por un carácter militante, vueltas hacia el objetivo práctico que siempre persiguió: hacer la revolución socialista.

En la China contemporánea, la realidad va más adelante que la teoria, aunque sin negarla, y proveé de los elementos necesarios para su avance. Trataremos de enunciar, punto por punto, en qué medida nos parece que la lógica de la historia $y$ del proceso revolucionario chino agotan hoy dia al socialismo como régimen de transición y comienzan a constituir un nuevo régimen, que no puede ser otro sino el comunista.

1. La revolución china, aunque su culminación haya sido el resultado de un proceso nacional, se produce en un cuadro internacional en que ya existe un bloque socialista. Aunque existieron contradicciones dentro de este bloque, su existencia fue fundamental para.el desarrollo del socialismo chino, ya sea desde el punto de vist:. de la protección contra una eventual agresión imperialista, ya sea por el hecho de que hasta 1960 proveyó al pueblo chino una ayuda técnica sustancial. A pesar de que hoy dia sus relaciones estén prácticamente rotas, la emergencia del comunismo en China está en parte posibilitada por el sistema internacional socialista, reconózcanlo o no los chinos. 
Esto confirma en alguna medida una de las tesis de los clásicos, de que el comunismo sólo seria posible en un régimen internacional, aunque haya que reconocer que los impases del socialismo en la uRss y su incapacidad hasta ahora de resolver sus problemas internos y avanzar también hacia al comunismo, dificulta en parte el avance chino, aunque sin lograr detenerlo.

2. China tenia una tradición cultural, quizás la más desarrollada del mundo, lo que posibilitó la continuación de su desarrollo aún después que la uRsS retiró su ayuda técnica. Los chinos ya habian desarrollado una tecnologia muy avanzada, y si occidente logró hacer la revolución industrial primero, fue sóla porque ellos no aplicaban esa tecnologia a la producción. Eso explica la sorpresa de los técniços soviéticos, cuando, después de decir que no habia condiciones para producir un aparato técnico, encontraban que pocos meses después ya estaba hecho el primero'. Eso explica también por qué, cuando utilizan como motivación el pensamiento del Presidente Mao, se verifican los "milagros" tan aclamados por los periódicos chinos. Claro es que China comercia con occidente e importa en alguna medida la tecnologia occidental; pero eso por si solo no explicaria su desarrollo industrial. Este, que se basa ahora sobre todo en el esfuerzo nacional, es un hecho que no puede ser negado simplemente porque los chinos no están interesados en desarrollar primordialmente una industria de bienes de consumo tipo occidental o porque no quieren provocar estimulos materiales (que entrarian en choque con los principios básicos de la sociedad que están tratando de crear). China empieza pues a tener un gran dominio de la tecnologia, que también era una de las condiciones apuntadas por los clásicos para lograr el comunismo.

3. China es por si sola un pais de dimensiones continentales, que tiene una gama muy variada de recursos humanos y materiales a ser explotados. Pero, tal vez por el hecho mismo de ser un pais hiperpoblado, para que pudiese proseguir su desarrollo y atender al mismo tiempo las necesidades de su población, tendria lógicamente que buscar que la organización social de la producción fuese la más efectiva y la más racional. Esto explica el nacimiento espontáneo de las comunas populares y el apoyo que tuvieron por parte del gobierno Cierto es, y los chinos lo reconocen, que en muchos puntos hasta hoy no lograron cumplir las metas propuestas. Pero el hecho es que las comunas son una forma superior de organización y que resuelven prácticamente muchos de los problemas vitales del desarrollo en China. La prueba más contundente de ello es que, si bien China no está todavia en una fase de abundancia, ya no existe hambre, y eso tiene que ser reconocido aún por sus más encarnizados enemigos. El desarrollo de las fuerzas productivas en China planteaba problemas que no podian encontrar respuesta en un régimen socialista y paradojalmente las condiciones para el avance estaban

\footnotetext{
${ }^{1}$ Karol cita varios casos ilustrativos de eso, por cjemplo el de la producción en Kunming, en 1957, de máquinas de precisión, p. 250 .
} 
dadas por aquellos factores que, en un primer momento, se presentaron como obstáculos; ss decir, las tradiciones culturales, el aislamiento relativo que China volvió a vivir después de igtoo, la sobrepoblación y la necesidad vital de proseguir el desarrollo, no sólo para atender a las necesidades de la población sino también para prepararse a una posible guerra con Estados Unidos. En esas circunstancias, no era ya posible mantener por mucho tiempo el principio socialista "a cada uno según su trabajo", sino que era necesario exigir de cada uno todo lo que pudiera dar según su capacidad y retribuirle sólo con lo que le era necesarió. Objetivamente, estaba planteada la necesidad de superar todos los obstáculos heredados del viejo orden, ya fuese desde el punto de vista material o del superestructural.

Pero en el socialismo, la superestructura ya comienza a adquirir un papel decisivo, en la medida en que es en ella donde están concentrados los más fuertes vestigios del viejo orden y es ella la que provee los principios de racionalización de los obstáculos materiales que sobreviven; Se hacian necesarias, pues, medidas que fuesen absolutamente radicales y que al mismo tiempo tuviesen una eficacia generalizada. Era necesario una revolución cuyos objetivos fuesen remover básicamente los obstáculos superestructurales que se oponian al nuevo régimen. Es ésta la explicación que nos parece más viable de la Gran Revolución Cultural. Nada puede tener de extraño, por tanto, que ésta se haya apoyado principalmente en la juventud; pues son las nuevas generaciones formadas ya en el espiritu comunitario (que las historias chinas para niños tan bien reflejan), las que tienen como tarea liquidar todos los vestigios de dominación y de capitalismo, haciendo avanzar al régimen hacia el comunismo. $\mathrm{Y}$ aunque el cáracter de esta revolución haya sido proletario no se opone a que - mientras existan vestigios de capitalismo-su enemigo principal sea el proletariado pues nadie puede encontrar raro que esa revolución no haya sido hecha por el proletariado especialmente, porque en la medida que se avanza hacia el comunismo, el proletariado como clase organizada y dirigente tiende a desaparecer y la especificidad de su papel se va disolviendo poco a poco dentro del conjunto social.

La burocracia, formada por el propio socialismo, va a ser el principal acusado de ese gran juzgado que es la Revolución Cultural, porque en aquélla se concentran los mayores obstáculos materiales y superestructurales para la implantación del nuevo orden.

Pero no se puede decir que fueron liquidados todos los obstáculos materiales, como tampoco puede afirmárse lo mismo de los culturales. Hay que comprender a ese proceso como uno del cual van emergiendo formas completamente nuevas de relaciones entre los hombres, que por demás no podria terminar en un plazo muy corto.

No obstante se podria indagar por qué se hizo con tanta amplitud una revolución cultural en lugar de tomar primero las medidas infraestructurales necesarias para los avances, si acaso es ésta la que condiciona aquélla. Aqui 
se plantea otro problema de suma importancia: la infraestructura es la condicionante en sociedades basadas en la explotación. En la medida en que esa explotación es golpeada a través de la liquidación de la propiedad privada y que el hombre va liberando su trabajo de la alienación, pasando a ser dueño de su destino, el condicionante comienza inmediatamente a ser la superestructura. Tal vez sea ésta una de las leyes del comunismo y en la medida que la Revolución Cultural tenga éxito se crearán las condiciones últimas para el avance incontenible de las fuerzas productivas.

Eș verdad que en toda situación revolucionaria la superestructura juega el papel fundamental. Pero cuando esta situación revolucionaria es la que inaugura un régimen comunista, se puede pensar que su papel fundamental no será nuevamente desplazado. En primer lugar, porque presupone ya un gran avance de las fuerzas productivas y de las relaciones de producción; en segundo lugar, porque el hombre estaria superando su prehistoria e iniciando su completo dominio, no sólo de la naturaleza, sino también de si mismo. Por lo tanto las ciencias en general, $y$ particularmente las ciencias sociales, serian la base superestructural fundamental sobre la cual el hombre montaria su civilización.

4. Cuando Marx decia que el comunismo aboliria la división entre el trabajo manual e intelectual, pensaba en un reino de la abundancia donde el trabajo manual, debido al desarrollo de la tecrologia, seria increiblemente reducido. Pára llegar a este punto en China queda mucho por recorrer; pero nos parece más lógico condicionar esto al comunismo que a la inversa. La realización de esto en una economia cápitalista es inconcebible dada la ausencia de planificación global y su carácter esencialmente anárquico. De este modo, la automatización generalizada no es, pues, condición para una sociedad igualitaria pero eso si, la presupone. Por otro lado, hay que comprender al comunismo como un proceso paulatino de cambio, que va desde sus formas iniciales más rudimentarias hàsta alcanzar sus caracteristicas más marcadas. La tesis de Marx era más bien una especulación sobre un problema que no estaba todavia planteado por la práctica.

Lo que se hace en China es tratar de liquidar esa división a través de la generalización de los dos tipos de trabajó Un intelectual es campesino u obrero una parte del año $y$ un campesino o un obrero es intelectual en la medida que trata de comprender el método marxista, si bien de forma simplificada, a través del pensamiento de Mao. Esas medidas en China son profundamente revolucionarias en el sentido de que intentan liquidar los status especificos, los papeles predeterminados, y posibilitan el despliegue hacia un efectivo igualitarismo. Trátase de un comunismo, quizás todavia en forma elemental, pero capaz de dar al mundo una gran demostración de efectividad.

Las implicaciones que esto acarrea para China son muy profundas. Lo 
que se pone en cuestión en un primer momento son los resabios de capitalismo; en un segundo momento se tienen que poner en jaque también los del socialismo. Hay que poner en duda la existencia misma del partido $y$ de todas las expresiones de poder hasta entonces vigentes. Tal vez por eso los chinos dicen que "la revolución cultural va a durar cien años".

\section{CHINA Y LA REVOLUCIÓN MUNDIAL.}

Todo este avance chino, sin embargo, no le garantiza el convertirse en lider de la revolución mundial. En primer lugar, porque si la uRss desempeñó durante un largo periodo ese papel, esto se debió fundamentalmente al hecho de haber sido la suya la primera revolución socialista. Hoy dia, todas las consecuencias negativas que acarré el stalinismo para los movimientos revolucionarios, constituyen lecciones que no se olvidarán. Ya han sido cuestionadas definitivamente las posibilidades del liderazgo de una gran potencia sobre los destinos de los movimientos revolucionarios; la autodeterminación de éstos tiende a ser un hecho.

En segundo lugar, porque aunque China en gran medida se ha mostrado capaz de oponer a los intereses nacionales los del movimiento revolucionario en otros paises - como muy bien lo muestra Karol en el último capitulo de su libro sobre la posición china en Vietnam, lass etapas distintas en que se encuentran el desarrollo de China y el de los paises que no hicieron aún su revolución, dificultan a menudo el diálogo. Además, en la orientación del movimiento revolucionario China cometió errores, como en el caso de Indonesia: También puede decirse que China intenta genẹralizar lo que sólo funciona bien allá, como por ejemplo la exagerada glorificación del Presidente Mao - que por más que se lọ respete, no deja de generar un cierto clima de malestar en el resto del mundo- en la medida en que recuerda la triste experiencia del stalinismo. Fuera de China no se comprende bien, en gran parte por culpa de los mismos chinos, que de hecho Mao es el' AntiStalin' por excelencia y que la divulgación de su pensamiento adopta esa forma únicamente por una cuestión de tradición milenar. Es verdad que la cultura china de hoy es radicalmente nueva pero todavia guarda mucho de las antiguas formas de presentarse al pueblo. Como dice Edgar Snow, se trata de "una nación regida por la moral, la ética, convencionalismos y filosofia". Si no se puede decir que el maoismo tenga hoy el mismo papel que tuvo el confucianismo en el pasado, por lo menos la forma como ambos se presentan son muy semejantes.

Pero de cualquier modo que sea, los profundos cambios que se operan en China tendrán consecuencias muy importantes en los movimientos revolucionarios de todo el mundo. Tal vez la más importante de ellas será la de mantener viva la llama del comunismo en medio de la crisis que vive el socialismo burocrático. 
Puede ser que todo el proceso que vive China actualmente no esté plenamente claro a nivel de la conciencia de los hombres que la dirigen; 0 puede ser que los chinos no tengan apuro en decir al mundo lo que están haciendo y estén esperando una mayor maduración de la situación. O puede que sea simplemente, por el mismo motivo por el que dejaron de divulgar sus estadisticas, el reflejo de un profundo desprecio por el juicio del resto del mundo. $O$ aún más, tal vez la convicción de que no serán comprendidos los lleve al rechazo de las posibilidades que permitan que sus ąsuntos internos sean puestos en tela de juicio. $O$, finalmente, puede ser que nosotros no hayamos logrado liberarnos de la visión occidental y que el análisis que hemos intentado hacer resulte equivocado. 